\title{
Announcement of New Editorial Policy
}

In view of the rapid diversification of the field of approximation theory and its applications, the editorial committee has decided to expand the scope of the journal.

Until recently, the central themes have included

1. approximation theory

2. numerical approximation

3. harmonic analysis

4. fractals and wavelets

Since Volume 19, Issue 1, the journal will focus on

(1) approximation theory

(2) computational analysis

(3) dynamic systems

(4) fractals

(5) function spaces

(6) geometric analysis

(7) harmonic analysis

(8) numerical approximation

(9) signal analysis

(10) special functions

(11) transform analysis

(12) wavelets

To accommodate this shift of emphasis, the name of this journal has been amended, replacing "Approximation" by "Analysis", and preserving the former title, "Theory and Applications". So the new name of "Approximation Theory and its Applications" is changed as "Analysis in Theory and Application" from 19:1, and still use "ATA".

The editors feel that approxiation theory, in particular, its approaches and methods in various branches of mathematics, plays an important role in a number of exciting new directions. Giving these proper emphasis on the above fields and related new fields will enhance the journal, and appeal to both the contributors and subscribers.

The format and printface have also been updated and more attractive to match the international prestige and readership. 